Giovani M. Zelada, MD

Internal Medicine-Psychiatry Residency

Program, University of Texas South-

western Medical Center, Dallas, TX
Paul B. Aronowitz, MD

Department of Internal Medicine,

University of California

Davis School of Medicine,

Sacramento, CA

\title{
Fever, rash, pruritus: Sweet syndrome
}

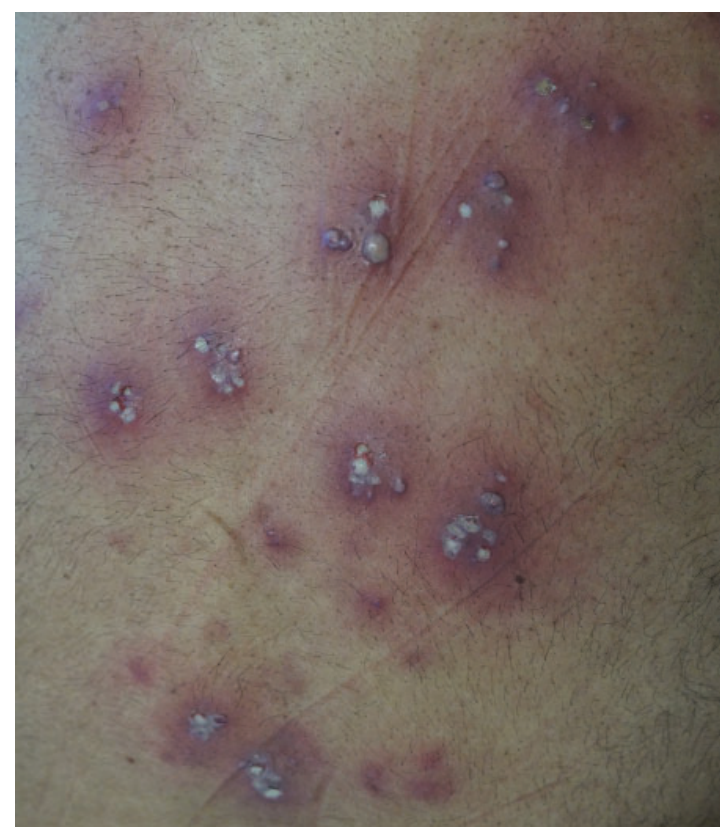

Figure 1. On the patient's back were multiple firm erythematous nodules with pustular heads, as well as several erythematous plaques.

40-YeAR-OlD man with a history of diaAbetes mellitus and hypertension, but taking no medications, presented to the hospital with a 5-day history of fever, rash, and pruritus. The patient initially presented to an urgent care center, where he received intramuscular and oral antibiotics, but his symptoms continued to worsen.

On physical examination, his temperature was $39.6^{\circ} \mathrm{C}$, heart rate 138 beats per minute, blood pressure 93/70 $\mathrm{mm} \mathrm{Hg}$, and respiratory rate 18 breaths per minute. He had elevated,

doi:10.3949/ccjm.88a.20053

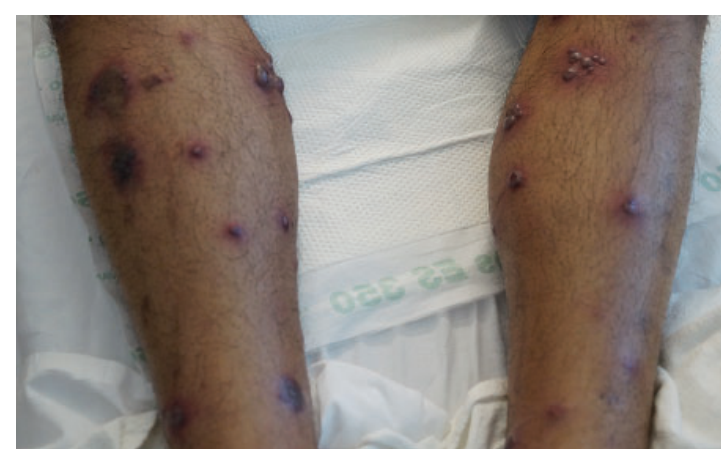

Figure 2. The patient's lower extremities.

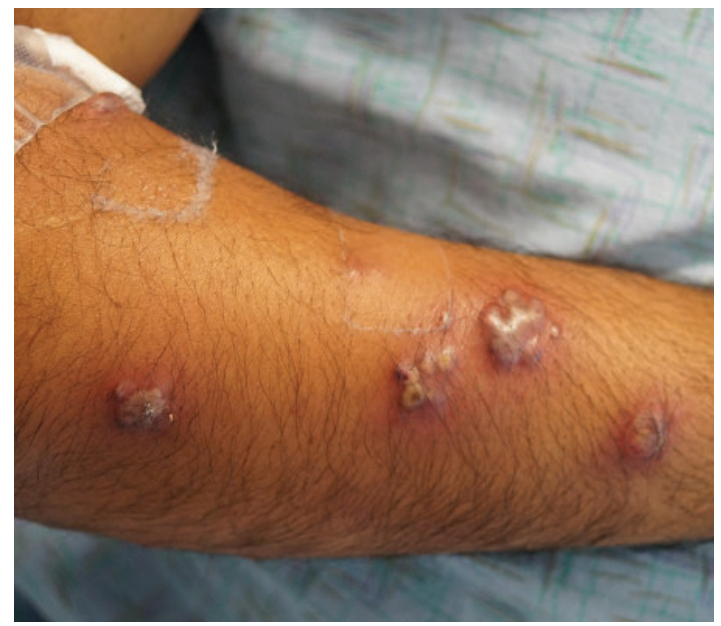

The patient presented with a 5-day history of fever, rash, and pruritus

tender, inflammatory plaques, nodules, and pustules distributed throughout his upper and lower extremities, back, chest, abdomen, scalp, and dorsal aspects of both feet (Figures 1-3).

His white blood cell count was $15.2 \times 10^{9} / \mathrm{L}$ (reference range $3.7-11.0 \times 10^{9} / \mathrm{L}$ ) with $60 \%$ neutrophils and $22 \%$ bands; the erythrocyte sedimentation rate was $48 \mathrm{~mm} /$ hour $(0-15$ $\mathrm{mm} /$ hour ), and the C-reactive protein level was $10.4 \mathrm{mg} / \mathrm{dL}(<0.9 \mathrm{mg} / \mathrm{dL})$. 


\section{TABLE 1}

\section{Diagnostic criteria for classic Sweet syndrome}

The diagnosis requires both major and minor criteria:

\section{Major criteria (both are required)}

Abrupt onset of painful erythematous plaques or nodules

Histopathologic evidence of a dense neutrophilic infiltrate without evidence of leukocytoclastic vasculitis

Minor criteria (2 of 4 are required)

Temperature $>38^{\circ} \mathrm{C}\left(100.4^{\circ} \mathrm{F}\right)$

Association with an underlying hemoproliferative disorder, inflammatory disease, or solid tumor

OR

Preceded by an upper respiratory or gastrointestinal infection

Excellent response to treatment with systemic corticosteroids or potassium iodide

Abnormal laboratory values at presentation (3 of 4 are required):

- Erythrocyte sedimentation rate $>20 \mathrm{~mm} / \mathrm{hour}$

- Elevated C-reactive protein

- White blood cell count > $8.0 \times 10^{9} / \mathrm{L}$

- Neutrophils $>70 \%$

Adapted from information in references 7 and 8 .

Because of concern for infectious dermatitis, his initial hospital treatment consisted of pain management, intravenous fluids, and intravenous vancomycin. However, a thorough infectious disease workup was unrevealing. Other considerations included a drug eruption, vasculitis, and various neutrophilic dermatoses (including pyoderma gangrenosum and Sweet syndrome). As a result, a skin biopsy was performed and revealed neutrophilic dermatitis without organisms, consistent with Sweet syndrome.

The patient began a 4-week course of prednisone in tapering doses $(60 \mathrm{mg}$ for 5 days, $40 \mathrm{mg}$ for 5 days, $20 \mathrm{mg}$ for 5 days, 10 $\mathrm{mg}$ for 5 days, then discontinued) and clobetasol $0.05 \%$ ointment twice daily for 7 days. $\mathrm{He}$ experienced rapid improvement of symptoms, which was noted during outpatient dermatology and primary care follow-up visits.

\section{SWEET SYNDROME: KEY FEATURES}

In 1964, R. D. Sweet, MD, first described acute febrile neutrophilic dermatosis, originally known as Gomm-Button disease, by its
4 cardinal features: fever; neutrophil polymorphonuclear leucocytosis of the blood; raised painful plaques on the limbs, face, and neck; and histologically, a dense dermal infiltration with mature neutrophil polymorphs, with no evidence of infection and prompt response to corticosteroids. ${ }^{1}$

There are 3 subtypes of Sweet syndrome:

- Classic or idiopathic

- Malignancy-related, most commonly hematologic but also observed in solid tumor malignancies ${ }^{2-5}$

- Drug-induced. ${ }^{1,6,7}$

In this patient's case, the abrupt appearance of fever, cutaneous lesions, and histopathologic findings, in the absence of malignancy or drug exposure and satisfaction of both major and 3 of 4 minor diagnostic criteria, were congruent with classic Sweet syndrome (Table 1). ${ }^{1,6-8}$ Notably, the patient did not have preceding symptoms suggestive of gastrointestinal or upper respiratory illness, was not on medications associated with Sweet syndrome, and never had signs or symptoms of hematologic or solid tumor malignancy.

The pathogenesis of Sweet syndrome is multifactorial, but studies have suggested a hypersensitivity reaction that promotes neutrophil activation ${ }^{9}$ and higher serum levels of granulocyte colony-stimulating factor ${ }^{10}$ leading to increased circulating neutrophils, both of which may explain this patient's leukocytosis with increased left shift.

Skin biopsy is the technical standard for diagnosis, but this patient's response to systemic steroids and resolution of leukocytosis (his initial white blood cell count of $15.2 \times 10^{9} / \mathrm{L}$ came down to $9.25 \times 10^{9} / \mathrm{L}$ over 4 days) also supports the typical response to treatment.

\section{STEROIDS ARE FIRST-LINE THERAPY}

Systemic corticosteroid therapy is the first-line treatment, but some case reports ${ }^{11,12}$ have also noted accelerated improvement in cutaneous lesions and reduced dependence on systemic steroids with adjunct topical glucocorticoid use, although the efficacy has not been formally studied.

Given their low potential for harm, topical steroids were used as adjuvant therapy, and the 


\section{ZELADA AND ARONOWITZ}

patient had rapid overall clinical improvement and resolution of his rash. Colchicine and potassium iodide are also considered first-line treatment choices. Indomethacin, cyclosporine, dapsone, and clofazimine have all been used as second-line treatments.

\section{DISCLOSURES}

The authors report no relevant financial relationships which, in the context of their contributions, could be perceived as a potential conflict of interest.

\section{REFERENCES}

1. Sweet RD. An acute febrile neutrophiic dermatosis. Br J Dermatol 1964; 76:349-356. doi:10.1111/j.1365-2133.1964.tb14541.x

2. Buck T, González LM, Lambert WC, Schwartz RA. Sweet's syndrome with hematologic disorders: a review and reappraisal. Int J Dermatol 2008; 47(8):775-782. doi:10.1111/j.1365-4632.2008.03859.x

3. Cohen PR, Kurzrock R. Sweet's syndrome and cancer. Clin Dermatol 1993; 11(1):149-157. doi:10.1016/0738-081x(93)90112-p

4. Cohen PR, Talpaz M, Kurzrock R. Malignancy-associated Sweet's syndrome: review of the world literature. J Clin Oncol 1988; 6(12):18871897. doi:10.1200/JCO.1988.6.12.1887

5. Nishie W, Kimura T, Kanagawa M. Sweet's syndrome evolved from recurrent erythema nodosum in a patient with myelodysplastic syndrome. J Dermatol 2002; 29(2):91-95. doi:10.1111/j.1346-8138.2002.tb00172.x

6. Anzalone CL, Cohen PR. Acute febrile neutrophilic dermatosis (Sweet's syndrome). Curr Opin Hematol 2013; 20(1):26-35. doi:10.1097/MOH.0b013e32835ad132

7. Cohen PR. Sweet's syndrome-a comprehensive review of an acute febrile neutrophilic dermatosis. Orphanet J Rare Dis 2007; 2:34. doi:10.1186/1750-1172-2-34
8. Walker DC, Cohen PR. Trimethoprim-sulfamethoxazole-associated acute febrile neutrophilic dermatosis: case report and review of drug-induced Sweet's syndrome. J Am Acad Dermatol 1996; 34(5 pt 2):918-923. doi:10.1016/s0190-9622(96)90080-8

9. Voelter-Mahlknecht S, Bauer J, Metzler G, Fierlbeck G, Rassner G. Bullous variant of Sweet's syndrome. Int J Dermatol 2005; 44(11):946-947. doi:10.1111/j.1365-4632.2004.02287.x

10. Kawakami T, Ohashi S, Kawa Y, et al. Elevated serum granulocyte colony-stimulating factor levels in patients with active phase of Sweet syndrome and patients with active Behcet disease: implication in neutrophil apoptosis dysfunction. Arch Dermatol 2004; 140(5):570-574. doi:10.1001/archderm.140.5.570

11. Cohen PR, Holder WR, Tucker SB, Kono S, Kurzrock R. Sweet syndrome in patients with solid tumors. Cancer 1993; 72(9):2723-2731. doi:10.1002/1097-0142(19931101)72:9<2723::aid-cncr2820720933>3.0.co;2-f

12. Cohen PR, Kurzrock R. Sweet's syndrome: a review of current treatment options. Am J Clin Dermatol 2002; 3(2):117-131. doi:10.2165/00128071-200203020-00005

Address: Paul Aronowitz, MD, MACP, Department of Internal Medicine, University of California, Davis, 4150 V Street, Suite 3100, Sacramento, CA 95817; paronowitz@ucdavis.edu 